\title{
A pair spectrometer for measuring multipolarities of energetic nuclear transitions
}

\author{
J. Gulyás ${ }^{\mathrm{a}}$, T.J. Ketel ${ }^{\mathrm{b}}$, A.J. Krasznahorkaya , M. Csatlós ${ }^{\mathrm{a}}$, L. Csige ${ }^{\mathrm{a}}$, Z. Gácsia ${ }^{\mathrm{a}}$, M. Hunyadia ${ }^{\mathrm{a}}$ A. Krasznahorkay ${ }^{\mathrm{c}}$, A. Vitéz $^{\mathrm{a}}$, \\ T.G. Tornyi ${ }^{\mathrm{a}}$
}

\author{
${ }^{a}$ MTA-ATOMKI, Institute for Nuclear Research, Hungarian Academy of Sciences \\ ${ }^{b}$ Nikhef National Institute for Subatomic Physics, Science Park 105, 1098 XG Amsterdam, The Netherlands \\ ${ }^{c}$ CERN,European Organization for Nuclear Research, Geneva, Switzerland
}

\begin{abstract}
in

A multi-detector array has been designed and constructed for the simultaneous measurement of energy-and angular correlations of electron-positron pairs. Experimental results are obtained over a wide angular range for high-energy transitions in ${ }^{16} \mathrm{O}$, ${ }^{12} \mathrm{C}$ and ${ }^{8} \mathrm{Be}$. A comparison with GEANT simulations demonstrates that angular correlations between 50 and 180 degrees of the $\mathrm{e}^{+} \mathrm{e}$ pairs in the energy range between 6 and $18 \mathrm{MeV}$ can be determined with sufficient resolution and efficiency.
\end{abstract}

Keywords: electron-positron pair spectrometer, internal pair conversion, multipolarity determination, anomalous angular correlation in ${ }^{8} \mathrm{Be}$

\section{Introduction}

Spectroscopy of internal pair conversion (IPC) has a long tra'dition [1, 2]. In a wide range of energies and atomic numbers, the conversion coefficient for internal electron-positron pair formation are fairly high, typically in the order of $10^{-4}-10^{-3}$ [3]. The measurement of these coefficients offers an effective method for determining the multipolarity of electromagnetic transitions (especially of high-energy and low-multipolarity transitions) [4].

The determination of the multipolarity of the high-energy transitions produced after particle capture reactions might be especially important for nuclear astrophysics to gain deeper un'derstanding of the dynamics of capture processes leading to a more accurate and reliable extraction of the astrophysical $S$ factor and the thermonuclear reactivity [5, 6].

In many light nuclei, the cross section for the radiative capture of protons, neutrons, deuterons and $\alpha$ particles has been observed to consist of a background slowly varying with beam 'energy, upon which the various known resonances in the reac"tion are superposed. This smooth background, which is important for nuclear astrophysics, has been identified as an extranuclear channel phenomenon, since the process takes place far from the nucleus (40-50 fm) rather than in the nuclear interior [7]. This has been designated as direct capture (single-step) reaction. The direct capture process represents a transition for the projectile from an initial continuum state to a final state via interaction with the electromagnetic field. Usually, it has a strong $E 1$ component but other multipolarities can also contribute. For the extraction and extrapolation of the astrophysical $S$ factor, it is important to know the multipole composition of such background radiations [5, 6].

Email address: kraszna@atomki.hu (A.J. Krasznahorkay)
The multidetector array is also designed to search for deviations from IPC due to the creation and subsequent decay into electron-positron pairs of a hypothetical short-lived neutral boson. Recent results from both underground and cosmic ray experiments suggest that dark matter may be explained by a light boson having a mass of $10 \mathrm{MeV}-10 \mathrm{GeV}$ and coupled to electrons and positrons. There have been several attempts to observe evidence for such particles, using data from running facilities [8-15] or re-analyzing old experiments [16-20]. Since no evidence for their existence was found, limits on its coupling to ordinary matter were set as a function of its mass. In the near future, new experiments are expected to extend those limits in a region of couplings and/or masses so far unexplored. It is not widely known, but indications were found for the existence of such a light boson also in some nuclear physics experiments. While anomaly was observed in the internal pair production, the overall results were not consistent with the involvement of a neutral boson [21, 22, 24]. A limit of $\leq 4.1 \times 10^{-4}$ was obtained for the boson to $\gamma$-ray branching ratio [21-25].

\section{Internal Pair Creation (IPC)}

Quantum electrodynamics (QED) predicts [1, 2] that the angular correlation between the $\mathrm{e}^{+} \mathrm{e}^{-}$pairs (emitted in IPC) peaks at $0^{\circ}$ and drops rapidly with the correlation angle $(\Theta)$ as shown in Fig. 1 .

The above calculations show that the angular correlations at small separation angles are almost independent of the multipolarity of the radiation, whereas at large separation angles, they depend critically upon the multipole order. Thus, it is important to measure angular correlations efficiently at large angles. 


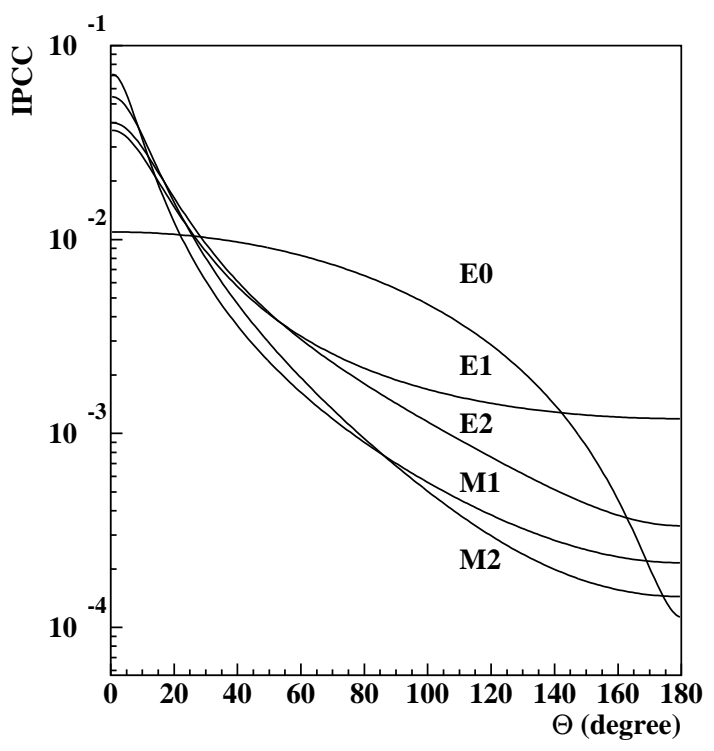

Figure 1: Calculated angular correlations of $\mathrm{e}^{+} \mathrm{e}^{-}$pairs obtained from IPC for different multipolarities and a transition energy of $\mathrm{E}_{\gamma}=17 \mathrm{MeV}$.

\section{The two-body decay of a boson}

When a nuclear transition occurs by emission of a short-lived $\left(\tau<10^{-13} \mathrm{~s}\right)$ neutral particle, the annihilation into an $\mathrm{e}^{+} \mathrm{e}^{-}$pair is anti-parallel (i.e. $\Theta_{c m}=180^{\circ}$ ) in the center of mass system. In the laboratory system, their angular distribution is peaked $\left(\Delta \Theta<10^{\circ}\right)$ at intermediate angles due to the Lorentz boost and provides an unique signature for the existence and a measure for the mass of an intermediate boson. In order to search for such an anomaly in the angular correlation, we need a spectrometer with sufficient angular resolution.

The invariant mass can be determined approximately from the relative angle $\Theta$ between $\mathrm{e}^{+}$and $\mathrm{e}^{-}$and from their energies in the following way[22]:

$$
m^{2} \approx\left(1-y^{2}\right) E^{2} \sin ^{2}(\Theta / 2)
$$

where $E=E^{+}+E^{-}+1.022 \mathrm{MeV}$ is the transition energy and $y=\left(E^{+}-E^{-}\right) /\left(E^{+}+E^{-}\right)$, with $E^{+(-)}$indicating the kinetic energy of the positron (electron) in the laboratory system.

\section{Overview of pair spectrometers}

Magnetic $\beta$ ray spectrometers were used first for internal pair formation studies [26-30]. Maximal detection efficiency of $10^{-4}$ for electron-positron pair detection was achieved for a few cases [28, 30]. Improvement of the pair resolution by improvement of the momentum resolution (to $1.3 \%$ ) with smaller particle transmission reduced the efficiency to $5 \times 10^{-6}$. An important advance [29] in the use of intermediate-image pair spectrometer was provided by the installation of a specially designed spiral baffle system which selected electron-positron internal pairs emitted at large relative angles $\left(50^{\circ} \leq \theta \leq 90^{\circ}\right)$.
The next generation of internal-pair spectrometers used two $d E / d x+E$ scintillator-detector telescopes for the detection of the electron-positron pairs in quadruple coincidence [31, 32]. A multi-detector (six scintillation electron telescopes plus an annular $\mathrm{Si}(\mathrm{Li})$ particle detector) high-efficiency pair spectrometer was built by Birk and co-workers [33]. An experimental pairline efficiency of $28 \%$ and a sum-peak energy resolution of $12 \%$ for the $6.05 \mathrm{MeV}$ E0 pair line in ${ }^{16} \mathrm{O}$ were achieved.

Schumann and Waldschmidt have detected internal pair spectra in the energy range of 2.8-6.5 MeV from an $(\mathrm{n}, \gamma)$ reaction with a combination super-conducting solenoid transporter plus $\mathrm{Si}(\mathrm{Li})$-detector spectrometer [34]. The pair-line efficiency of the spectrometer [35] was large, but it had a very limited discrimination power for different multipolarities in this energy region.

The Debrecen superconducting solenoid transporter plus two-Si(Li)-detector electron spectrometer was also adapted for internal-pair studies [36]. The observed pair-line efficiency for two detectors operated in sum-coincidence mode was $35 \%$, while the energy resolution was $0.6 \%$ at $2 \mathrm{MeV}$. A similar spectrometer built by Kibédi and co-workers [37] and has been used recently for internal pair studies [38].

A highly segmented phoswich array of plastic scintillators was constructed for measurements of $\mathrm{e}^{+} \mathrm{e}^{-}$pairs emitted in high-energy electromagnetic transitions in nuclei by Montoya and co-workers [39]. Electron (positron) energies of 2-30 MeV can be measured by each individual element, with a total transition energy resolution of $\delta \mathrm{E} / \mathrm{E}=13 \%$ for a $20 \mathrm{MeV}$ transition. The array covers $29 \%$ of the full solid angle and its efficiency is $1.6 \%$ for a $6 \mathrm{MeV} E 0$ internal pair decay, and $1.1 \%$ for an 18 $\mathrm{MeV} E 1$ transition.

A positron-electron pair spectroscopy instrument (PEPSI) was designed to measure transitions in the energy region of 10$40 \mathrm{MeV}$ by Buda and co-workers [40]. It consists of $\mathrm{Nd}_{2} \mathrm{Fe}_{14} \mathrm{~B}$ permanent magnets forming a compact $4 \pi$ magnetic filter consisting of 12 positron and 20 electron mini-orange-like spectrometers.

A $\Delta E-E$ multi-detector array was constructed by Stiebing and co-workers [41] from plastic scintillators for the simultaneous measurement of energy and angular correlation of $\mathrm{e}^{+} \mathrm{e}^{-}$ pairs produced in internal pair conversion (IPC) of nuclear transitions up to $18 \mathrm{MeV}$. The array was designed to search for deviations from IPC stemming from the creation and subsequent decay into $\mathrm{e}^{+} \mathrm{e}^{-}$pairs of a hypothetical short-lived neutral boson. The angular resolution of the spectrometer determined by the solid angle of the telescopes was $\Delta \Theta=15^{\circ}$, while the efficiency for one pair of telescopes: $\approx 3 \times 10^{-5}$. The investigated angular range was extended from $20^{\circ}$ to $131^{\circ}$.

In this paper, we present a novel $\mathrm{e}^{+} \mathrm{e}^{-}$pair spectrometer equipped with multi-wire proportional chambers and large volume plastic scintillator telescopes having remarkably higher efficiency and better angular resolution than previously obtained by Stiebing and co-workers [41]. 


\section{Monte-Carlo simulations}

Monte Carlo (MC) simulations of the experiment were performed using the GEANT3 code in order to determine the detector response function. For different transition energy and multipolarity a lookup table is created for electron an positron energies and correlation angle using the Rose calculations [2]. The first electrons (or positrons) are generated isotropically, with $\phi_{e}$ random between 0 and $2 \pi$ and $\theta_{e}$ as a sine distribution, and the second particles with relative angles $\phi$ and $\theta$, with $\theta$ according to the lookup table. Isotropic emission of pairs would also result in a sine distribution for the relative angles $\theta$, the so-called correlation angle.

Also boson decays can be generated as well as gamma ray coincidences. The electrons and positrons are followed through the setup and the detected energy losses are stored, including detection of annihilation radiation from the stopped positrons. The energy loss steps are small until a final energy of $90 \mathrm{keV}$. The simulated events are stored in a similar way as the measurements, but now as precise deposited energies and positions inside the wire chambers and including the generated electron and positron energies and correlation angles.

\section{The spectrometer}

Plastic scintillator detectors combine reasonable energy resolution with minimum response to $\gamma$ radiation and with excellent characteristics for fast, sub-nanosecond coincidence timing, which is crucial for good background reduction. Thus, we use plastic $\Delta \mathrm{E}-\mathrm{E}$ detector telescopes for the detection of the $\mathrm{e}^{+} \mathrm{e}^{-}$. In contrast to Ref. [41], very thin $\Delta E$ detectors $(52 \times 52 \times 1$ $\mathrm{mm}^{3}$ ) were chosen that gives a remarkably improved $\gamma$ suppression. The $E$ detectors have similar dimensions $(80 \times 60 \times 70$ $\mathrm{mm}^{3}$ ) as in Ref. [41]. The spectrometer setup is shown in Fig. 2 with six scintillation detector telescopes and six position sensitive gaseous detectors at 60 degrees relative to their neighbors surrounding the target inside the carbon fiber beam pipe. The response of the detector set-up as a function of correlation angle theta for isotropic emission of e+e- pairs is shown in Fig. 2 (bottom). A detector with $4 \pi$ solid angle acceptance would show a sine distribution and the simulated curve with three sharp peaks can be understood as the limited phase space with only detector combinations at 60, 120, and 180 degrees with an angular range in a single detector of about 40 degrees. Another setup with five telescopes will be also described with a smoother acceptance for the angular correlation of the $\mathrm{e}^{+} \mathrm{e}^{-}$ pairs.

$\gamma$ rays were detected by a Ge clover detector at a distance of $25 \mathrm{~cm}$ from the target behind the Faraday-cup. The detector has an active volume of $470 \mathrm{~cm}^{3}$ and it is also equipped with a BGO anti-coincidence shield [42].

The positions of the hits are measured by multiwire proportional counters (MWPC) which was constructed at ATOMKI based on the concept of Ref. [43] and placed in front of the $\Delta E$ and $E$ detectors. The anode of the MWPC is a set of parallel $10 \mu \mathrm{m}$ thick gold-plated tungsten wires at a distance of 2
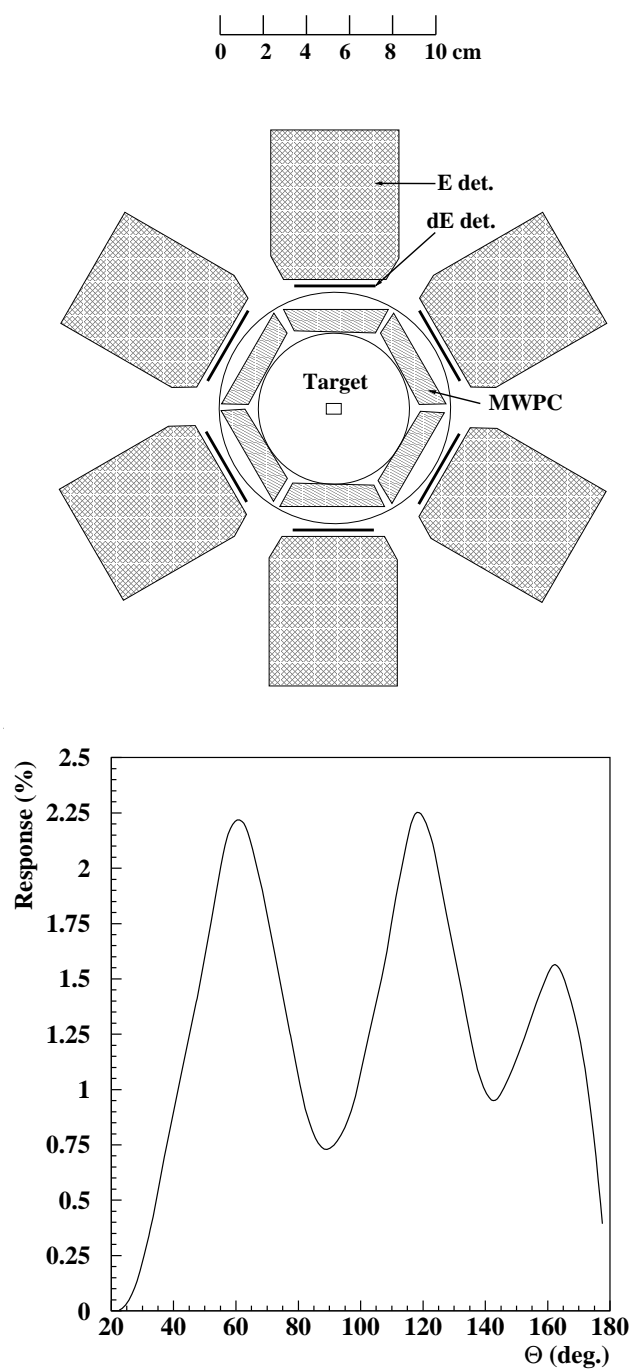

Figure 2: (top) Initial arrangement with six telescopes and (bottom) detection response as a function of the correlation angle between the $\mathrm{e}^{+} \mathrm{e}^{-}$pairs in Monte Carlo simulations.

$\mathrm{mm}$ from each other. The two cathodes are composed of silverplated copper wires having a diameter of $0.1 \mathrm{~mm}$ and separated by $1.27 \mathrm{~mm}$. The anode-cathode distance is $3.5 \mathrm{~mm}$. The two cathodes are placed perpendicularly to each other giving the $x$ and $y$ coordinates of the hit. Delay-line read-out (10 ns/taps) is used for the cathode wires. $\operatorname{Ar}(80 \%)+\mathrm{CO}_{2}(20 \%)$ counting gas was flowing across the detector volume at atmospheric pressure. The accuracy of the $(x, y)$ coordinates implies an angular resolution of $\Delta \Theta=2^{\circ}(\mathrm{FWHM})$ in the $40^{\circ}-180^{\circ}$ range, which is approximately five times better than in Ref. [41]. The efficiency of the MWPC detectors was estimated to be $80 \%$.

\subsection{Beam and Target}

To minimize the amount of material around the target, a 24 $\mathrm{cm}$ long electrically conducting carbon fiber tube with a radius of $3.5 \mathrm{~cm}$ and a wall thickness of $0.8 \mathrm{~mm}$ is used. The target, positioned perpendicular to the beam, is mounted on a target holder supported from the back by two perspex rods of $3 \mathrm{~mm}$ diameter. The original $0.5 \mathrm{~mm}$ thick Al target holders 
with $10 \mathrm{~mm}$ inner diameter opening were replaced when data showed shadowing due to scattering in the aluminum sides. The GEANT simulations confirmed this shadowing and also some background via external pair production. To avoid this, the targets were evaporated onto 10-micron thick, 50-mm long and $5 \mathrm{~mm}$ wide Al strips, which was stretched between two 3-mm thick Plexiglas rods. The rods are arranged parallel to the beam and their distance from the beam was $25 \mathrm{~mm}$. On the basis of simulations, they did not cause significant background via external electron-positron conversion. The bars were 12-cm long, and the placement was done so as not to cause any shadowing effects in any of the telescopes. The targets have a typical thickness of $0.3 \mathrm{mg} / \mathrm{cm}^{2}$, which is adapted to the resonance width of the reaction under investigation as well as to the demand of a sufficient real-to-random ratio of coincidences. The beam is absorbed in a Tantalum Faraday-cup $15 \mathrm{~cm}$ behind the target.

\subsection{Trigger for data readout and data-acquisition}

The signals from the photomultipliers of the $\Delta \mathrm{E}-\mathrm{E}$ detectors are processed in constant fraction discriminator units (CF8000). The CFD thresholds are adjusted slightly above the noise level of the $\Delta \mathrm{E}$ detectors (which are essentially insensitive to $\gamma$ ray events) and a bit higher for the $E$ detectors. Chance events from double (or multiple) hits by $\gamma$ rays in the $E$ detectors are suppressed by requiring a $\Delta \mathrm{E}-\mathrm{E}$ coincidence. The resulting telescope signals are analyzed by a logical unit requiring multiplicity- 2 coincidences. In order to allow the simultaneous measurement of single telescope events, the trigger module is set to allow a scaled-down fraction of single telescope events as well. Time and energy signals of the $\Delta \mathrm{E}-\mathrm{E}$ detectors as well as the time signals (Up, Down, Left, Right) of the MWPC detector are recorded. The spectra of single telescope events are used for on-line monitoring of the efficiencies and an approximate energy calibration of the $E$ detectors. Especially for the $\Delta \mathrm{E}$ detectors with their low CFD thresholds this on-line survey is important. In the off-line analysis these spectra provide a reliable way to determine the telescope efficiencies.

\subsection{Energy calibration of the spectrometer}

The energy calibration of the telescopes for low energies was made with the Compton edges of a ${ }^{60} \mathrm{Co}$ source, while at high energies we used the Compton edges of high energy $\gamma$ transitions coming from proton capture reactions. The high energy edges of the singles electron spectra offered also good calibration points, which was used for on-line gain monitoring and corrections as well. It was possible to correct the gain shifts with a precision of about $1 \%$, well below the energy resolution of the detectors. Finally, the sum energy spectra was checked for the $6.05-\mathrm{MeV}$ transition in ${ }^{16} \mathrm{O}$ excited in the ${ }^{19} \mathrm{~F}(\mathrm{p}, \alpha){ }^{16} \mathrm{O}$ reaction, the $4.44-\mathrm{MeV}$ and $15.1-\mathrm{MeV}$ transitions in ${ }^{12} \mathrm{C}$ excited in the ${ }^{11} \mathrm{~B}(\mathrm{p}, \gamma){ }^{12} \mathrm{C}$ reaction and the $17.6 \mathrm{MeV}$ line in ${ }^{8} \mathrm{Be}$ excited in the ${ }^{7} \mathrm{Li}(\mathrm{p}, \gamma)^{8} \mathrm{Be}$ reaction.

The energy threshold settings of the $\Delta \mathrm{E}$ detectors were found to be also very important. We had to make sure that we are not cutting too much from the low-energy part of the energyloss distributions. The energy calibration of those detectors was based on the comparison of the measured and simulated energy loss distributions of the strong 6.05 MeV EO transition in ${ }^{16} \mathrm{O}$. An example of such a measurement is shown in Fig. 3

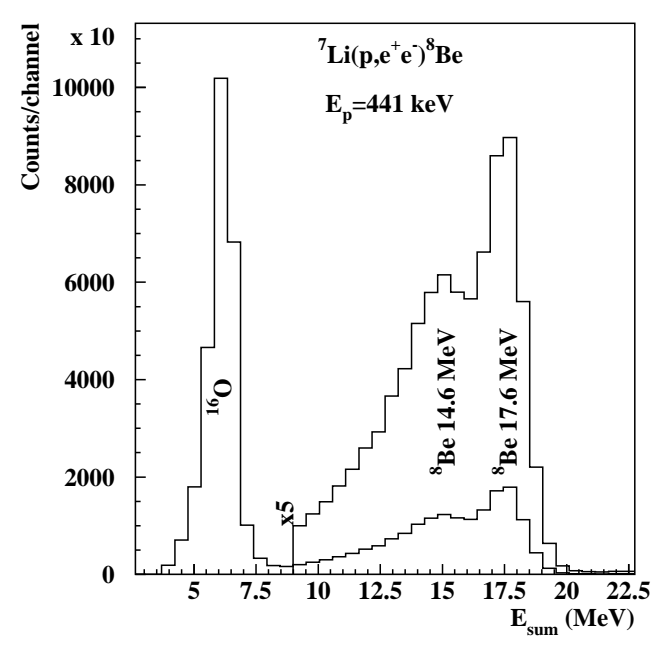

Figure 3: Total energy spectrum, reconstructed from the sum energy deposited in the scintillators, the undetected energy loss and the mass of the $\mathrm{e}^{-} \mathrm{e}^{+}$pairs, produced at $\mathrm{E}_{p}=0.441 \mathrm{MeV}$ using $\mathrm{LiF}_{2}$ targets.

\subsection{Efficiency calibration of the spectrometer}

It was crucial for the precise angular correlation measurements to measure and understand the response to isotropic $\mathrm{e}^{+} \mathrm{e}^{-}$ pairs of the whole detector system as a function of the correlation angle. We were aiming at a precision of about $1 \%$ for the shape of the response function.

The detectors measure continuous $\mathrm{e}^{+} \mathrm{e}^{-}$spectra and the sum of the energies are constructed off-line. Due to the energy loss in the wall of the chamber and in the $\Delta \mathrm{E}$ detectors, as well as the finite thresholds of the discriminators (CFD), the low-energy part of the spectrum is always cut out. Thresholds should be set equally to have similar efficiencies for the different telescopes. After a proper energy calibration of the telescopes, it was done by software cuts. The response of the MWPC detectors depends slightly on the position of the hit, the energy of the particle and might slowly change also in time.

The response curve depends primarily on the geometrical arrangement of the detector telescopes. As shown in Fig. 2, initially we used six equivalent telescopes placed symmetrically around the target. However, due to the six-fold rotational symmetry of the spectrometer and the finite solid angle of the detectors, the response varied drastically as a function of the correlation angle. Moreover, at the minima of the curve, the edge effects of the detectors dominated, which made the response values under-defined. Thus, it was advantageous to break the rotational symmetry to make the response curve smoother. Since we also had to increase the response around 90 degrees, we set the geometry of the setup as shown in Fig. 4.

Beside the $\mathrm{e}^{+} \mathrm{e}^{-}$coincidences, the down-scaled single electron events ( $\Delta E-E$ coincidences) were also collected during 


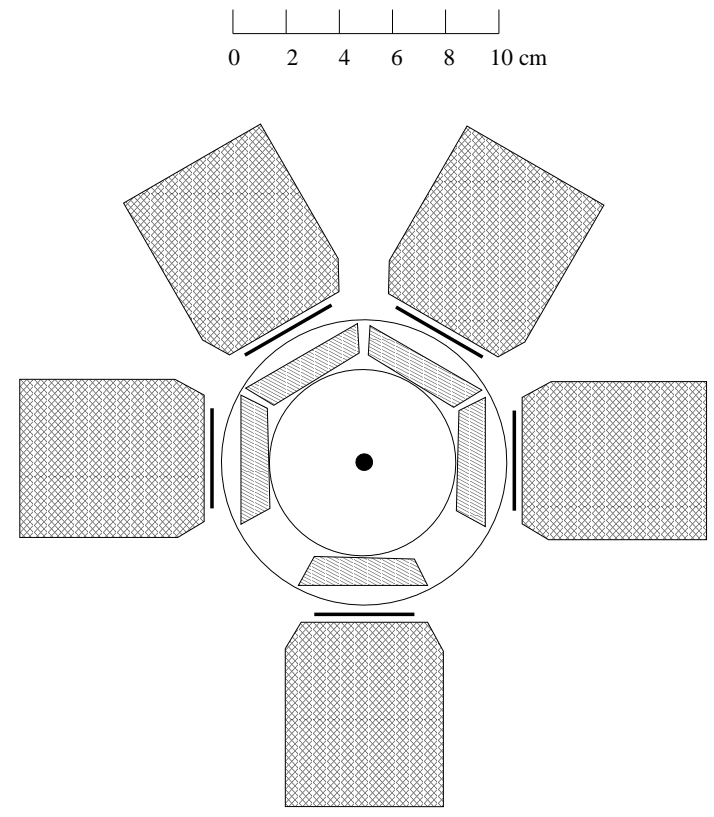

Figure 4: Final schematic arrangement with five telescopes to detect $\mathrm{e}^{+} \mathrm{e}^{-}$pairs.

the whole experiment for making experimental energy and response calibrations. An event mixing method [44] was used to determine experimentally the relative response of the spectrometer as a function of the correlation angle. According to the method, uncorrelated lepton pairs were generated from subsequent single events and their correlation angle was calculated as for the coincident events. The resulted angular correlation for the uncorrelated events gave us the experimental response curve. Reasonably good agreement was obtained with the results of the MC simulations as presented in Fig. 5.

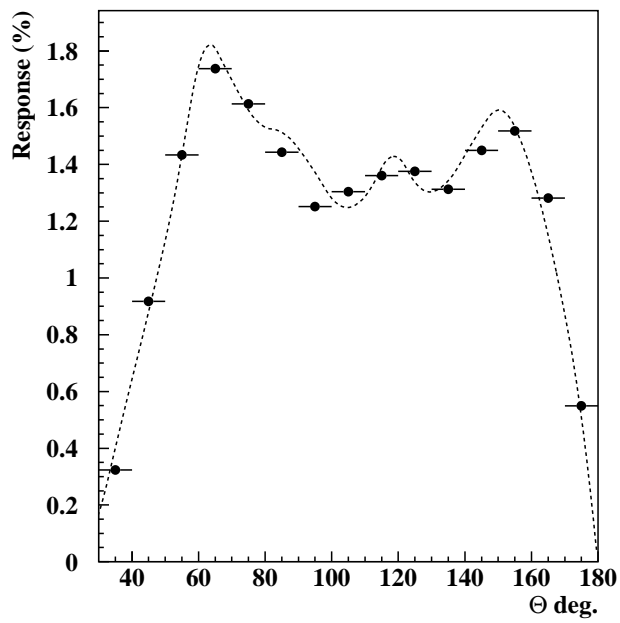

Figure 5: Detector response for the five-telescope setup as a function of correlation angle $(\theta)$ for isotropic emission of $\mathrm{e}^{+} \mathrm{e}^{-}$pairs (curve) in Monte Carlo simulations and (data points) from experimental data as explained in the text.

When electrons from the target pass through the set-up to the wire chambers multiple scattering in the target holder, in the wall of the carbon fiber vacuum chamber, and in the wire chamber windows takes place. This gives rise to an angular spread of the reconstructed angular correlation.

The simulated angular resolution corresponds to FWHM $\approx 7$ degrees. We use bins of 10 degrees in the correlation spectra.

The shape of the coincidence response curve depends also on position of the beam spot, which may walk during a long experiment. However, using the above event mixing method, this effect can be compensated, so the extracted angular correlation will be independent of small variations in the beam spot position.

In order to check the experimentally determined response curve with data, the angular correlation of the $\mathrm{e}^{+} \mathrm{e}^{-}$pairs created in the $6.05 \mathrm{MeV} \mathrm{E0}$ transition was measured and corrected by the response curve determined in the same experiment. As shown in Fig. 6 very good agreement has been obtained with the theoretically predicted $E 0$ angular correlation.

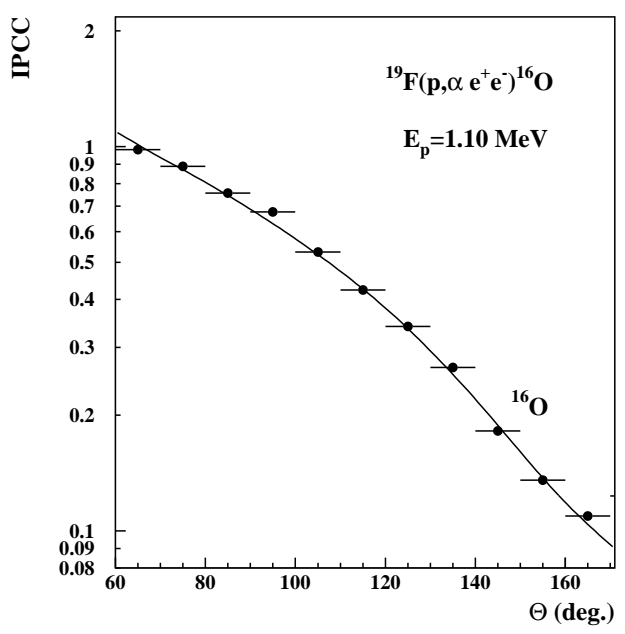

Figure 6: Angular correlation of the $\mathrm{e}^{+} \mathrm{e}^{-}$pairs that originated from the ${ }^{16} \mathrm{O}$ $6.05 \mathrm{MeV} E 0$ transition excited in the ${ }^{19} \mathrm{~F}(\mathrm{p}, \alpha){ }^{16} \mathrm{O}$ reaction at $\mathrm{E}_{p}=1.10 \mathrm{MeV}$ compared with the $\mathrm{MC}$ simulation assuming pure $E 0$ transition.

\subsection{Background by cosmic muons}

Cosmic muons going through the spectrometer produce coincidences between the $\Delta E-E$ telescopes and the MWPC detectors, similarly to $\mathrm{e}^{+} \mathrm{e}^{-}$pairs. We measure low coincidence rates, especially at large separation angles, so the effect of traversing cosmic rays has to be considered. Background measurements have been performed before and after the experiments with the settings (gates, thresholds, etc.) of the in-beam measurement, and the angular correlation of the background events were subtracted with a weighting factor. This factor was determined by comparing the high energy part $\left(E_{\text {sum }}>20 \mathrm{MeV}\right)$ of the sum energy spectra measured in-beam and off-beam, which contained only cosmic events in both cases. 


\section{Measured pure $E 1$ and $M 1$ transitions}

To demonstrate the reliability of the spectrometer, we investigated a pure $E 1$ transition in ${ }^{12} \mathrm{C}$ and and a pure $M 1$ transition in ${ }^{8} \mathrm{Be}$ as well. The ${ }^{12} \mathrm{C}$ resonance at $17.2 \mathrm{MeV}$ with a width $\Gamma=1.15 \mathrm{MeV}$ is populated in the ${ }^{11} \mathrm{~B}(\mathrm{p}, \gamma){ }^{12} \mathrm{C}$ reaction at $1.6 \mathrm{MeV}$ bombarding energy. It decays by isovector $E 1$ transitions to the ground state and first excited state with energies of 17.2 and $12.8 \mathrm{MeV}$. The ${ }^{8} \mathrm{Be}$ resonance at $17.6 \mathrm{MeV}$ with $\Gamma=11 \mathrm{keV}$ is populated in the ${ }^{7} \mathrm{Li}(\mathrm{p}, \gamma){ }^{8} \mathrm{Be}$ reaction at $441 \mathrm{keV}$ proton bombarding energy. It decays to the ground state and the particle-unstable first excited state $(\Gamma=1.5 \mathrm{MeV})$ with 17.6 and 14.6 MeV isovector $M 1$ transitions.

Figure 7 shows the angular correlations for the above $M 1$ and $E 1$ transitions compared with the simulated full curves which confirms the reliability of our setup. We have not observed significant anomaly for the $17.6 \mathrm{MeV}$ isovector $M 1$ transition in ${ }^{8} \mathrm{Be}$ reported earlier [21].

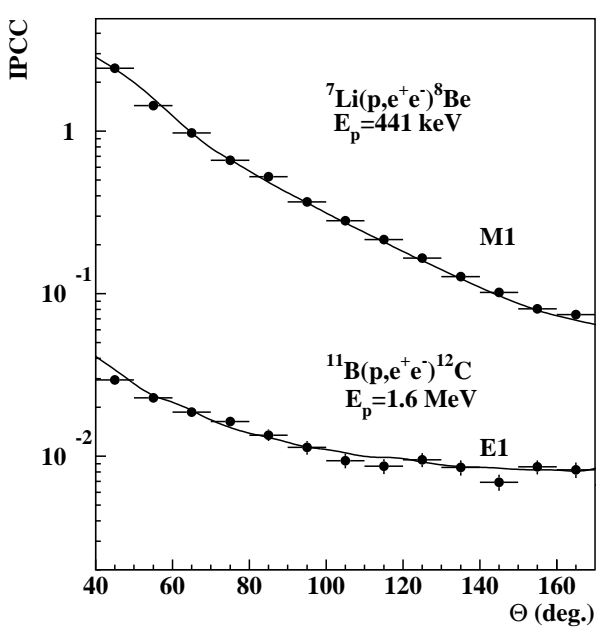

Figure 7: Measured and simulated angular correlations for $\mathrm{e}^{+} \mathrm{e}^{-}$pairs stem from a typical $E 1\left({ }^{12} \mathrm{C} 17.2 \mathrm{MeV}\right)$ and $M 1\left({ }^{8} \mathrm{Be} 17.6 \mathrm{MeV}\right)$ transitions showing the very large discrimination power of the IPCC for determining multipolarities. The values are rescaled for better comparison of the shapes.

\section{Outlook}

We repeated the experiment in order to investigate another $M 1$ transition in ${ }^{8} \mathrm{Be}$, which is isoscalar in contrast to the 17.6 $\mathrm{MeV}$ transition. The ${ }^{8} \mathrm{Be}$ resonance at $18.1 \mathrm{MeV}(\Gamma=168 \mathrm{keV})$ was populated in the ${ }^{7} \mathrm{Li}(\mathrm{p}, \gamma){ }^{8} \mathrm{Be}$ reaction at $1.030 \mathrm{MeV}$ proton bombarding energy. It decays to the ground state and the particle unstable first excited state with 18.1 and $15.1 \mathrm{MeV}$ isoscalar M1 transitions.

We have observed significant $(5 \sigma)$ deviation at $\Theta \approx 135^{\circ}$ from the simulated angular correlation in the case of the 18.1 $\mathrm{MeV}$ isoscalar $M 1$ transition in ${ }^{8} \mathrm{Be}$ with branching ratio relative to $\gamma$ ray emission of $\approx 5 \times 10^{-6}$. It can not be explained by any $E 1$ admixture coming from the direct capture process. It has disappeared below and above the $18.1 \mathrm{MeV}$ resonance.
However, it can be explained by the creation and decay of a light $\left(m_{0} c^{2}=16.7 \mathrm{MeV}\right)$ isoscalar $\mathrm{J}^{\pi}=1^{+}$boson [45].

\section{Acknowledgements}

We are deeply indebted to Fokke W. N. de Boer, who proposed to search for a short-lieved neutral boson in ATOMKI already in 2000. Together with him we performed many challenging experiment in Debrecen. Fokke sadly passed away in 2010. This paper is dedicated to his memory. We are indebted also to Kurt Stiebing for making his spectrometer available for our early experiments. This work has been supported by the Hungarian OTKA Foundation No. K106035, by the European Community FP7 - Capacities, contract ENSAR n ${ }^{\circ} 262010$ and by the European Union and the State of Hungary, co-financed by the European Social Fund in the framework of TÁMOP4.2.4.A/2-11/1-2012-0001 National Excellence Program.

\section{References}

[1] R. Wilson, Internal Pair Formation, in: K. Siegbahn (Ed.), Alpha-, betaand gamma-ray spectroscopy, Vol. 2, North-Holland Publishing Company, Amsterdam, 1965, Ch. XXV(c), p. 1557.

[2] M.E. Rose, Phys. Rev. 76 (1949) 678. M.E. Rose, Phys. Rev. 78 (1950) 184.

[3] P. Schlüter and G. Soff, At. Data Nuct. Data Tables 24 (1979) 509.

[4] P. Schlüter, G. Soff, W. Greiner, Phys. Rep. 75(6) (1981) 327.

[5] R.M. Chasteler et al., Physical Review Letters, 72 (1994) 3949.

[6] M. Spraker et al., Phys. Rev. C61 (1999) 015802.

[7] C. Rolfs, Nuclear Physics A217 (1973) 29.

[8] H. Merkel, et al., Phys. Rev. Lett., 106, 251802, (2011).

[9] S. Abrahamyan, Phys. Rev. Lett., 107, 191804 (2011).

[10] J. P. Lees et al., Phys. Rev. Lett. 108, 211801 (2012).

[11] B. Echenard, Mod. Phys. Lett. A 27, 1230016 (2012).

[12] F. Archilli et al., Phys. Lett. B706, 251 (2012).

[13] D. Babusci et al., Phys. Lett. B720, 111 (2013).

[14] P. Adlarson et al., Phys. Lett. B726, 187 (2013).

[15] R. Barnabei et al., Phys.Rev. D 77 (2008) 023506; http://arxiv.org/pdf/1412.6524.pdf

[16] J. D. Bjorken, R. Essig, P. Schuster, and N. Toro, Phys. Rev. D 80, 075018 (2009).

[17] S. Andreas, C. Niebuhr, and A. Ringwald, Phys. Rev. D 86, 095019 (2012).

[18] J. Blumlein and J. Brunner, Phys. Lett. B701, 155 (2011).

[19] S. N. Gninienko, Physical Review D, 85, 055027, (2012).

[20] S. N. Gninienko, Phys. Lett. B713, 244 (2012).

[21] F.W.N. de Boer et al., Phy. Lett. B 388, 235 (1996).

[22] F.W.N. de Boer et al. J. Phys. G 23, L85 (1997).

[23] M.J. Savage, B.W. Filippone, L.W. Mitchell, Phys. Rev. D37 (1988) 1134.

[24] F.W.N. de Boer et al. J. Phys. G 27 L29 (2001).

[25] D.R. Tilley et al., Nucl. Phys.A745, 155 (2004).

[26] H. Daniel and W. Bothe, Naturforschung 90, 402 (1954).

[27] R. Bent. T. Bonner and R. Sippel, Phys. Rev. 98, 1237 (1955).

[28] D.E. Alburger, Rev. Sci Instr. 27 (1956) 991. DE. Alburger, Phys. Rev. 1111586 (1958).

[29] E.K. Warburton, D.E. Alburger, A. Gailman, P. Wagner and L.F. Chase, Jr., Phys. Rev. 133 (1964) B42.

[30] J. Kjetlman and B. Jvhans, Ark. Fysik 14, 17 (1958).

[31] J.C. Adloff, K.H. Souv, D. Diadier, F. Scheibling, P. Chevallier aud Y. Wolfson, Phys. Rev. C 10, 1819 (1974).

[32] M. Ulrickson, N. Benczer-Koller. J.R. MacDonald aud J.W. Tape, Phys. Rev. C15, 186 (1977).

[33] M. Birk, J.S. Sokolowski, Y. Wolfson and N. BenczerKoller, Nuci. Iristr. and Meth. 203, 255 (1982).

[34] S. Schumann and M. Waldachmidt, Z. Physik 271, (1974) 97 (1974).

[35] M. Watdschnaidt and P. Osterman, Nucl. Instr. and Meth. 8965 (1970). 
[36] A. Passoja, P. Tikkanen, A. Krasznahorkay, Z. Gácsi, T. Kibédi and T. Fényes, Nucl . Instr. and Meth. 223, 96 (1984).

[37] T. Kibédi et al., Nucl . Instr. and Meth. A294, 523 (1990).

[38] T. Kibédi et al., EPJ Web of Conferences 3506001 (2012); (DOI: 10.1051/epjconf/20123506001).

[39] Montoya et al., Nucl . Instr. and Meth. A334 437 (1993).

[40] A. Buda, J.C.S. Bacelar, A. Balanda, J . van Klinken, Z. Sujkowski and A. van der Woude, Nucl . Instr. and Meth. A335, 479 (1993).

[41] K.E. Stiebing et al., J. Phys. G 30, 165 (2004).

[42] Z. Elekes et al., Nucl. Instr. and Meth. 503, 580 (2003).

[43] G. Charpak, F. Sauli, Nucl. Instr. and Meth. 162, 405 (1979).

[44] S. Ravan et al., Phys. Rev. C89, 024906 (2014).

[45] A.J. Krasznahorkay et al., To be submitted to Phys. Rev. Lett. 